\title{
SURFACE MICROMACHINED POLYSILICON THERMAL ACTUATOR ARRAYS AND APPLICATIONS
}

\author{
John H. Comtois and Victor M. Bright \\ Air Force Institute of Technology \\ Department of Electrical and Computer Engineering \\ Wright-Patterson AFB, OH 45433-7765
}

\begin{abstract}
Thermal actuator arrays have been developed to complement the capabilities of electrostatic actuators. This paper demonstrates how arrays of two or more thermal actuators can be applied to a variety of basic building-block micromechanical devices such as stepper motors, optical component positioners, and grippers.
\end{abstract}

\section{INTRODUCTION}

Electrostatic actuators can operate at low power and high frequencies, but they require close tolerances or high voltages to achieve large forces, and typically have small deflections. Surface micromachined polysilicon thermal actuators are capable of large deflections and high forces in a current/voltage regime that is compatible with standard integrated circuit electronics. The devices presented in this paper were designed and fabricated in polysilicon using the ARPA-sponsored Multi-User MEMS Process, but could be re-created in any MEMS process that provides at least one releasable, current carrying layer.

\section{ACTUATOR DESIGN}

Thermal expansion is a strong force, but a single-material actuator based solely on the thermal expansion of a beam would have a small deflection. The actuators presented in this paper use a deflection multiplying structure based on a 'thermo-magnetic' actuator originally demonstrated in the LIGA process [1]. A typical ' $U$ ' shaped lateral thermal actuator or 'heatuator' is shown in Figure 1. Current is passed through the actuator, and the higher current density in the narrower 'hot' arm causes it to heat and expand more than the wider 'cold' arm. The arms are joined at the free end, which constrains the actuator tip to move laterally in an arcing motion towards the cold arm side.

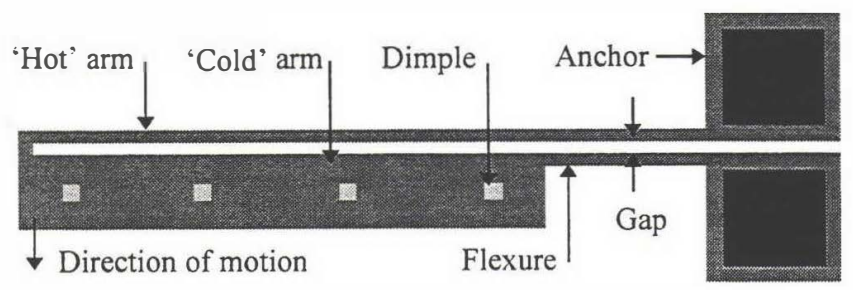

Figure 1. Basic layout of the lateral thermal actuator. Typical dimensions are: 'hot' arm $2.5 \mu \mathrm{m}$ wide, $240 \mu \mathrm{m}$ long; 'cold' arm $16 \mu \mathrm{m}$ wide, $200 \mu \mathrm{m}$ long; flexure $2.5 \mu \mathrm{m}$ wide, $40 \mu \mathrm{m}$ long, and gap $2 \mu \mathrm{m}$ wide. The $4 \mu \mathrm{m}$ square dimples prevent stiction. Deflections of $16 \mu \mathrm{m}$ can be achieved at $3 \mathrm{~V}$ and $3.5 \mathrm{~mA}$ in $2 \mu \mathrm{m}$ thick polysilicon.

The only requirement to achieve lateral motion with this actuator structure is to have one arm expand more than the other. So the actuator design can take on a wide range of geometries, including actuators with vertical motion or external heating. Close tolerances are not required, and this extremely simple design can be fabricated in any MEMS process that includes at least one releasable, current carrying layer such as SCREAM, $i$ MEMS ${ }^{n}$, and many other proprietary technologies. The thermal actuators presented in this paper were fabricated in the ARPA-sponsored Multi-User MEMS Process (MUMPS) [2].

Structural features which increase the temperature difference between the hot and cold arms will increase the efficiency of the actuator. The simplest method is to make the cold arm wider or taller to decrease the current density or increase the surface area for cooling. Other investigators have shown that a trench etched under the hot arm of a metal actuator increased the thermal isolation from the substrate, thereby decreasing the heat lost from the hot arm and increasing the deflection versus current ratio [3].

Polysilicon has a higher resistivity than metal, which allows the actuator to operate in a voltage and current regime more closely matched to standard integrated circuit electronics. For example, a $220 \mu \mathrm{m}$ long, $2 \mu \mathrm{m}$ thick actuator with a $2.5 \mu \mathrm{m}$ wide hot arm produced a force of $4.4 \mu \mathrm{N}$ at an input power of $10.8 \mathrm{~mW}$, delivered at $2.94 \mathrm{~V}, 3.68 \mathrm{~mA}$, which is completely compatible with standard CMOS circuitry. These actuators can deflect up to $16 \mu \mathrm{m}$ at the tip when unloaded, and the $4.4 \mu \mathrm{N}$ force was delivered at an $8 \mu \mathrm{m}$ deflection. Isolated actuators will operate at maximum deflection up to $300 \mathrm{~Hz}$, and in one particular optical application, were observed to operate up to $1 \mathrm{kHz}$ when driven to a $1.75 \mu \mathrm{m}$ deflection [4]. Other details of actuator testing and design considerations have been presented elsewhere [5].

These actuators exhibit a back-bending characteristic when driven past the point where they stop deflecting in the forward direction. The mechanism is a deformation of the hot arm which shortens it. When the current is removed, the actuator is left permanently back-bent from its originally fabricated position. The amount of back-bending depends on the amount of over-driving. Large back-bending deflections are slightly less predictable than forward deflections, but small amounts of back-bending can be controlled [5]. This permanent back-bend of an unpowered actuator is useful for assembling and positioning MEMS devices.

In forward deflection against a stiff structure, a long, thin hot arm may bow rather than push; but in back-bending the hot arm is in tension, so it can deliver more force. For example, the $220 \mu \mathrm{m}$ long actuator mentioned above can deliver $15.5 \mu \mathrm{N}$ when backbending. To back-bend that actuator, $7 \mathrm{~V}, 5.8 \mathrm{~mA}$ was applied for 5 seconds, but this is a one-time power requirement. After backbending the actuator will operate as before, but now starting from a 'negative' deflection relative to its original position. This is a beneficial design option, as the actuator applications will show.

\section{ACTUATOR ARRAYS}

A longer polysilicon actuator is capable of greater deflection and force, but the trade-offs are that the electrical resistance 
increases with the actuator's length, as does chance of the device failing due to stiction; and a longer hot arm is likely to deliver less force due to bowing. A more practical approach to obtaining higher forces is to connect several actuators with a common structure: a flexible yoke attached perpendicularly at the free ends of parallel thermal actuators, with flexures to accommodate their arc-like motion. Other options for arraying lateral thermal actuators are possible, but the yoke concept has proven to be the most compact, both with a single row of actuators and two rows of actuators set opposite to each other. An added benefit of arraying two or more actuators opposite to each other is that the yoke cancels out their arcing and expanding motions, producing purely linear motion, in addition to combining their forces. An array of ten actuators with a yoke can be seen on the right side of Figure 2.

With the yoke design, the force of the array can be increased by simply adding actuators to the same yoke. Since each actuator has its own attaching flexure, adding more actuators does not increase the stiffness of the yoke. Arrays of 60 actuators on a single yoke have been successfully operated, and more could easily be added. The actuators in the arrays shown below are wired in parallel. There was some initial concern over current sharing through so many parallel paths, but the resistance of the polysilicon increases with temperature, providing built-in current leveling. Any actuator which draws more current will heat more, and as its resistance increases its current draw will decrease.

An important consideration for using these arrays is that they produce a larger deflection when they have been given an initial back-bend. This allows the actuators to move through their zero position, where the least amount of force is lost in flexing the yoke and tethers. Provision must be made at the point where the array is attached to a driven mechanism if back-bending is to be used.

\section{APPLICATIONS}

The following applications are presented as examples of how these actuator arrays can be applied to a variety of basic buildingblock micromechanical devices such as stepper motors, optical component positioners, and grippers. The first application uses an actuator array to position and modulate a hinged mirror in a corner cube retroreflector (CCR). A CCR has three mutually perpendicular, mirrored walls. This mirror arrangement will reflect light back in the direction of its incoming path, and is commonly used in roadside reflectors. The device was proposed as a possible MEMS communication link [6], though no design specifics were given. A recently reported design uses hinged polysilicon plates for the mirrors [7]. Designs developed at the Air Force Institute of Technology (AFIT) took a similar hingedmirror approach, but with electrostatically or thermally actuated mirrors.

The thermally actuated design, shown in Figure 2, has a static gold mirror on the substrate and two hinged, gold-plated mirrors. One hinged mirror is positioned and modulated with a thermal actuator array. The notched end of the array tether slides into a keyhole when the plate is lifted. The tether end can have several notches to set the initial angle of the plate. The other hinged mirror is held by a slotted locking plate designed to position the mirror within $3.5 \mathrm{mrad}$ of perpendicularity with the substrate. The hinged mirror plates are made as thick as possible to counter stress in the gold layer, keeping the mirror flat. They are constructed of two releasable polysilicon layers with a layer of silicon dioxide trapped between them, for a total thickness of $4.25 \mu \mathrm{m}$ [8].

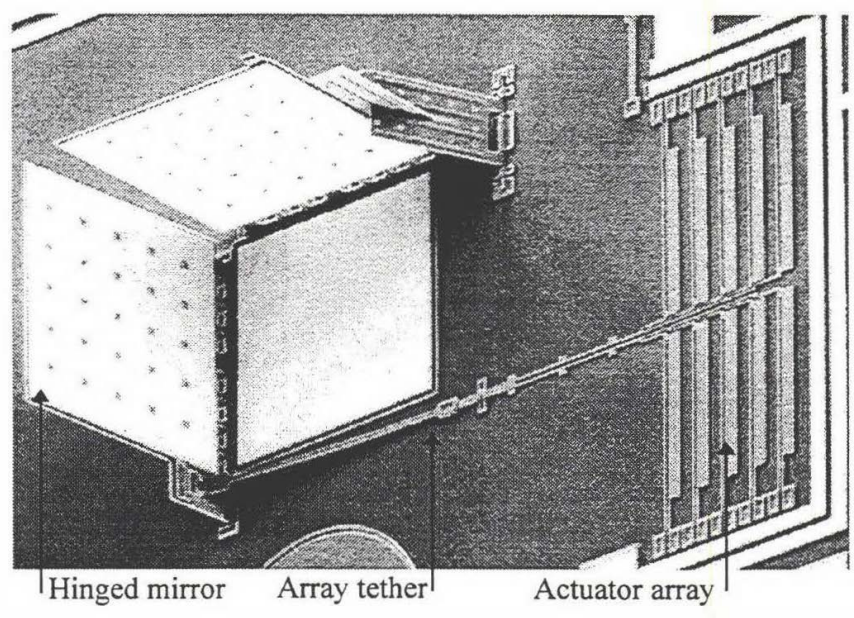

Figure 2. Corner Cube Retroreflector with an actuator array to position and modulate a hinged plate mirror. Mirrors are $280 \mu \mathrm{m}$ square, actuators are $240 \mu \mathrm{m}$ long, $20.5 \mu \mathrm{m}$ wide, and $2 \mu \mathrm{m}$ thick.

The next application is a gripper design, shown in Figure 3. The actuators are slid over the edge of the die after fabrication and overhang the die edge by $350 \mu \mathrm{m}$. Electrical connection to the actuator pair is made via $19 \mu \mathrm{m}$ wide hinged-wires. These create gold-on-gold contacts with only $5.5 \Omega$ of contact resistance each, which is small compared to the $645 \Omega$ resistance of the actuators. The wires are $1.5 \mu \mathrm{m}$ thick polysilicon with $0.5 \mu \mathrm{m}$ thick gold on top. When they are flipped over, the gold on top is now on the bottom, where it shorts across gold contacts on the gripper frame and the substrate wires.

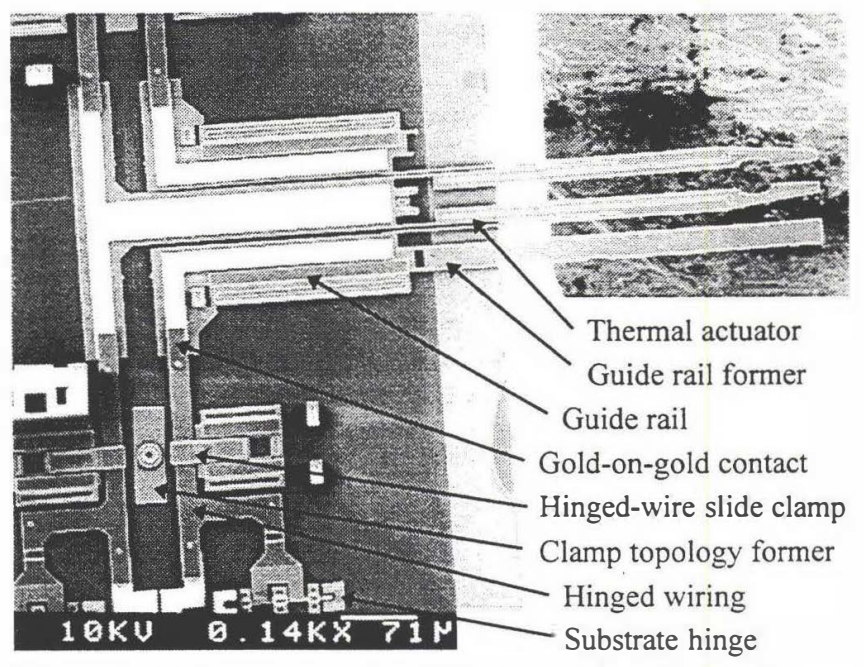

Figure 3. Grippers over edge of the die and wired with flip-over hinged-wires. Clamps hold wires in tension against gripper and substrate wiring. Topology former for the sliding clamps rotates out of the way. Gripper closes a full $16 \mu \mathrm{m}$ at $2.7 \mathrm{~V}, 3.3 \mathrm{~mA}$. 
The wires are held in place by clamps whose topology is shaped by removable formers [8] to allow enough space under the clamp face to easily slide over the wire. The formers are rotated out of the way before the wires are flipped over. The wires are flexed to provide the spring force to make low resistance contacts, but they must be pressed down manually for the clamp to slide over them. The gripper frame also has formers to give the guide rails the proper topology so the frame can slide under them. These formers can be removed after the grippers are assembled; one is shown removed in Figure 3.

A method of increasing the deflection of the thermal actuator arrays is to build them into rotary or linear stepper motors. Figure 4 shows a rotary stepper motor with a $200 \mu \mathrm{m}$ diameter rotor. A main array of ten $240 \mu \mathrm{m}$ long thermal actuators is attached to a drive pawl, which is engaged and disengaged from the rotor by a second array of three pusher actuators set at $90^{\circ}$ to the main array. This arrangement of actuators leaves much of the rotor edge free to connect to other structures such as gear trains or cam levers.

Fewer actuators can be used; motors with a single pusher and two drive actuators have been operated. The force delivered through the rotor has not been measured, but similar actuator arrays have been measured delivering $1.6 \mu \mathrm{N}$ of force per actuator when forward bending, and $3.5 \mu \mathrm{N}$ per actuator when backbending against a static beam. Therefore, the amount of force delivered through the rotor will depend on the number of actuators in the main array, and whether the motor is being run clockwise or counterclockwise.

The actuators in this motor are operated from a slightly backbent position, as shown in Figure 4. The back bending appears as a slight bow in the hot arms of the actuators which, due to the current leveling property of these actuators, all get the same amount of bend. For example, on this motor the pusher array was back-bent with a $5 \mathrm{sec}$ application of $7.5 \mathrm{~V}$, but operates at $3.7 \mathrm{~V}$.

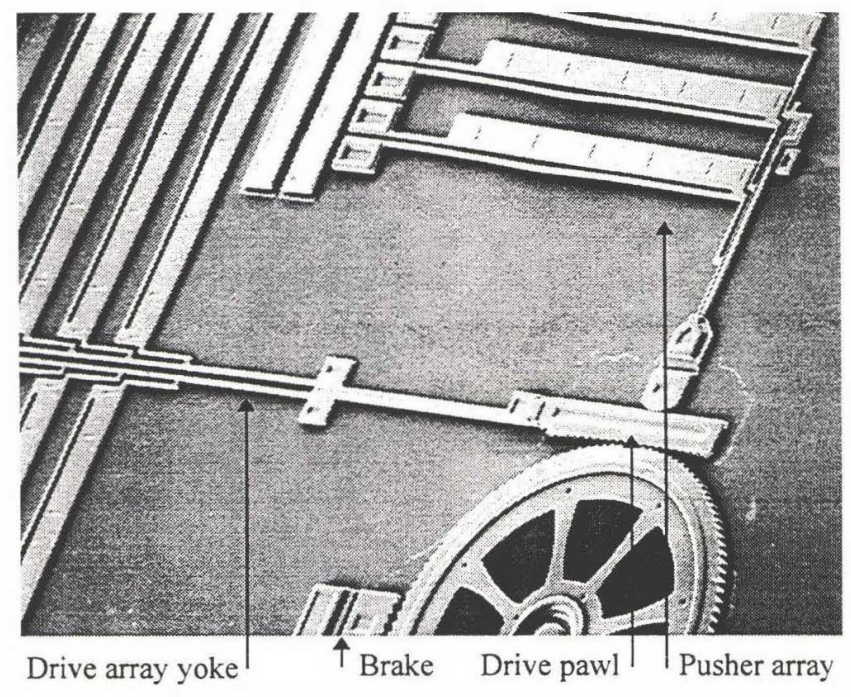

Figure 4. Rotary stepper motor with thermal actuator arrays back-bent into their operating position, with the drive pawl engaged. The three pusher actuators that engage the drive pawl. and the ten actuators that move the rotor are $240 \mu \mathrm{m}$ long, $21 \mu \mathrm{m}$ wide, and $2 \mu \mathrm{m}$ thick. The rotor is $200 \mu \mathrm{m}$ in diameter.
The pusher array is back-bent to place the drive pawl in contact with the rotor, which is fabricated in the disengaged position. The pawl is left in the engaged position as a brake when no power is applied. A second brake attached to a single actuator is also available for holding the rotor in position while the drive pawl is disengaged, in case there is a backwards force from the structure being driven by the motor. The drive pawl length allows variation in the amount of main drive back bend.

One important detail is the thickness of the teeth on the pawls and rotor. In the MUMPS process it is possible to create $3.5 \mu \mathrm{m}$ thick structures by stacking the two releasable polysilicon layers [8]. The extra thickness prevents the pawls from sliding over the rotor when driven. The drive pawl can also be tethered directly to the pusher array with a thin flexure to prevent slide-over.

For testing, the actuator arrays were sequenced with a digital timing circuit which operates reed relays to apply power to the actuators. A typical timing sequence for driving the motor of Figure 4 counter-clockwise is shown in Figure 5. This circuit can drive the arrays up to $200 \mathrm{~Hz}$; so with the 64 teeth on the rotor being engaged two at a time, the motor will rotate at $375 \mathrm{rpm}$. For positioning optical components, the device to be positioned can be placed directly on the rotor as shown in Figure 6, a device currently in fabrication, or the rotor can be geared to the device.

\begin{tabular}{|l|llll}
\hline Drive & Retracting & Stopped & Pulling & Stopped \\
\hline & & & \\
\hline Push & Disengaged & & Engaged \\
\hline & & & \\
\hline Brake & Brake on & Brake off \\
\hline
\end{tabular}

Figure 5. Typical timing sequence for driving the rotary stepper motor of Figure 4 in the counter-clockwise direction.

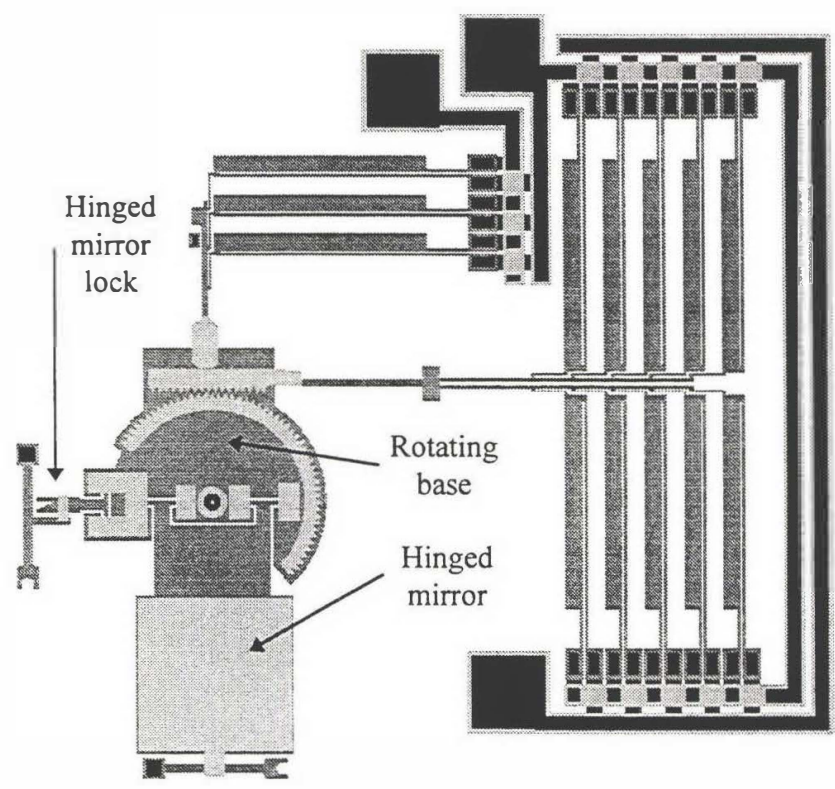

Figure 6. Rotary stepper motor built directly into a hinged, rotating mirror base. Motor could also be geared to the base to provide finer positioning increments. The base is $200 \mu \mathrm{m}$ across. 
The sarne two-array actuation scheme can be applied to linear motors by replacing the rotor with a sliding, toothed rack. Figure 7 shows a linear stepper motor driven by one-sided arrays. Another configuration which takes up less space uses one pair of arrays which push the rack against a stationary wall to engage it. In the test motor of Figure 7 the rack can be moved $200 \mu \mathrm{m}$, but for applications such as sliding grippers off the die, the rack could be as long as needed and incorporated in the device being positioned.

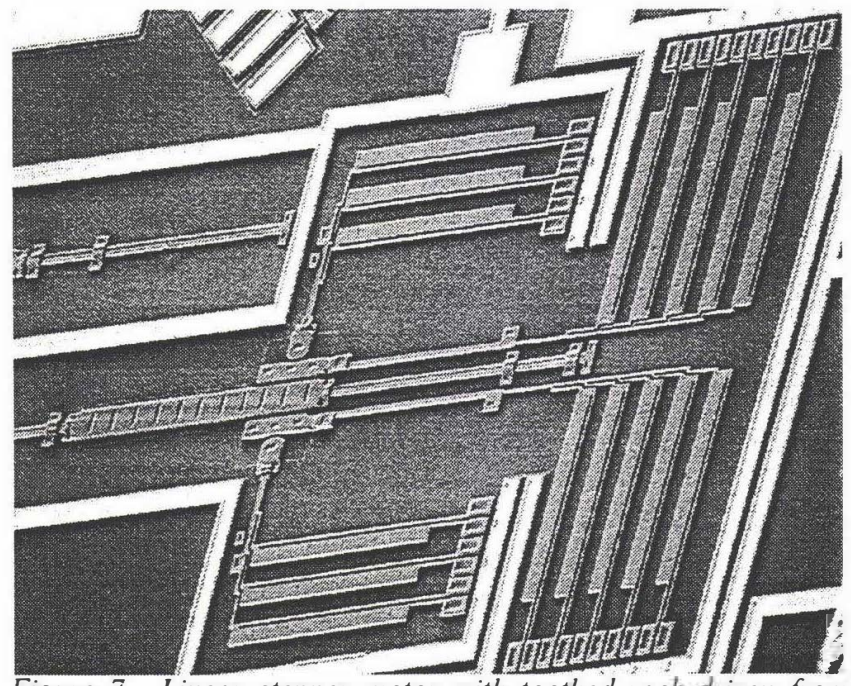
Figure 7. Linear stepper motor with toothed rack driven from both sides by arrays of $240 \mu \mathrm{m}$ long, $2 \mu \mathrm{m}$ thick actuators. The rack on this motor can slide $200 \mu \mathrm{m}$.

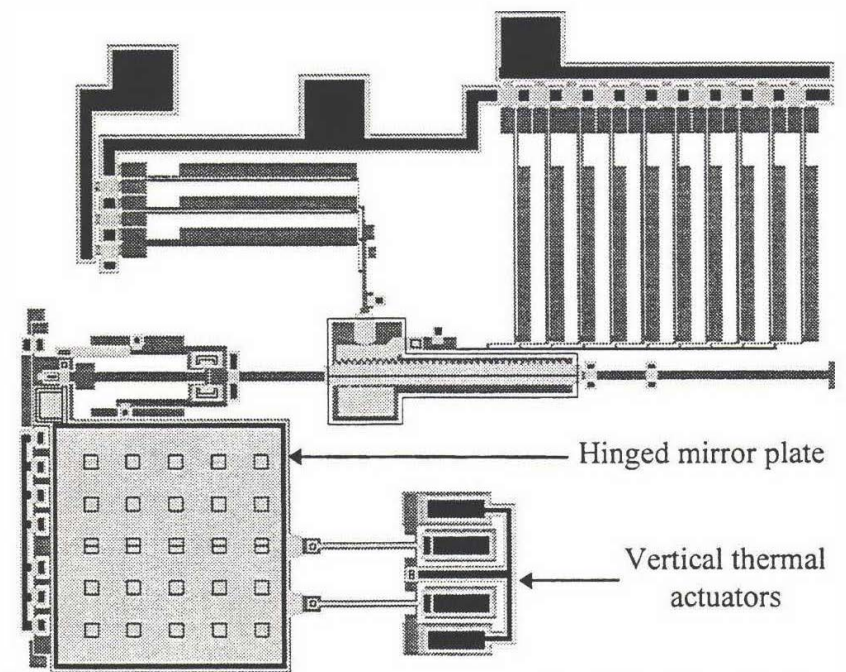

Figure 8. Linear stepper motor arranged to lift a hinged mirror plate for self-assembly of optical devices. Vertical thermal actuators provide the initial lift.

Another potentially useful application of stepper motors is the self-assembly of hinged structures. Such structures are typically assembled manually, a time-consuming and delicate process. Thermally actuated stepper motors have sufficient force to raise and position hinged plates. Figure 8 shows the layout of a motor designed to lift a hinged mirror. In this design, currently in fabrication, vertically acting thermal actuators provide an initial lift to prevent the linear stepper motor from pushing against the hinges without lifting the plate.

\section{CONCLUSION}

These applications demonstrate how thermal actuator arrays can expand the microelectromechanical actuator design space. Their high force, large deflections, and compatibility with standard integrated circuit voltages complement the characteristics of electrostatic actuators, and make them ideal choices where compact actuators with high forces or deflections are of greater concern than their power consumption and operating frequency. Their simple design and modest lithography and fabrication requirements mean they could be fabricated in any MEMS process that includes a releasable, current carrying layer.

\section{ACKNOWLEDGEMENT}

This work was sponsored by the USAF Phillips Laboratory PL/VTEE, Kirtland AFB, New Mexico.

\section{REFERENCES}

1. H. Guckel, J. Klein, T. Christenson, K. Skrobis, M. Laudon, and E. Lovell, "Thermo-magnetic Metal Flexure Actuators," Technical Digest, Solid State Sensors and Actuators Workshop, Hilton Head, SC, 13-16 June 1992, pp. 73-75.

2. D. Koester, R. Mahedevan, and K. Marcus, Multi-User MEMS Processes (MUMPS) Introduction and Design Rules, rev. 3, Oct 1994, MCNC MEMS Technology Applications Center, 3021 Cornwallis Road, Research Triangle Park, NC, 27709.

3. L. Field, D. Buriesci, P. Robrish, and R. Ruby, "Micromachined 1x2 Optical Fiber Switch," Proc. Transducers '95/Eurosensors IX, Stockholm, Sweden, 1995, pp. 344-347.

4. D. Sene, J. Grantham, V. Bright, and J. Comtois, "Development and Characterization of Micro-mechanical Gratings for Optical Modulation," Proc. IEEE MEMS-96 Workshop, San Diego, CA, 11-15 Feb. 1996, pp. 222-227.

5. J. Comtois, V. Bright, and M. Phipps, "Thermal Microactuators for Surface-micromachining Processes," Proc. SPIE: Micromachined Devices and Components, 23-24 October 1995, vol. 2642, pp. 10-21.

6. K. Brendley, and R. Steeb, "Military Applications of Microelectromechanical Systems," RAND report to the Office of the Secretary of Defense, United States Air Force, United States Army, RAND, Santa Monica, CA, 1993.

7. D. Gunawan, L. Lin, and K. Pister, "Micromachined Corner Cube Reflectors as a Communication Link," Sensors and Actuators A, 46-47, pp. 580-583, 1995.

8. J. Comtois and V. Bright, "Design Techniques for SurfaceMicromachining MEMS Processes," Proc. SPIE: Micromachining and Microfabrication Process Technology, vol. 2639, 23-24 Oct. 1995, pp. 211-222. 